\title{
BMJ Open Psychological distress and type 2 diabetes mellitus: a 4-year policemen cohort study in China
}

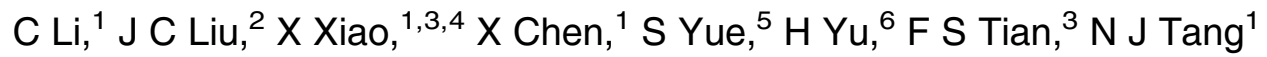

To cite: Li C, Liu JC, Xiao X, et al. Psychological distress and type 2 diabetes mellitus: a 4-year policemen cohort study in China. BMJ Open 2017;7:e014235.

doi:10.1136/bmjopen-2016014235

- Prepublication history and additional material is available. To view please visit the journal (http://dx.doi.org/ 10.1136/bmjopen-2016014235).

$\mathrm{CL}$ and JCL contributed equally to this work and should be considered co-first authors

Received 10 September 2016 Revised 19 November 2016 Accepted 7 December 2016

CrossMark

For numbered affiliations see end of article.

Correspondence to

Dr N J Tang, Department of Occupational and

Environmental Health, School of Public Health, Tianjin

Medical University, NO. 22,

Qixiangtai Road, Heping

District, Tianjin 300070,

China; tangnaijun@tmu.

edu.cn

$\mathrm{OR}$

Dr F S Tian

fengshitian080101@126.com

\section{ABSTRACT}

Objectives: This study investigated whether psychological distress predicts the development of type 2 diabetes mellitus (T2DM) and if the association differs between populations at a high or low diabetes risk level among Chinese police officers.

Design: Prospective cohort study.

Setting: Single centre.

Participants: 6559 participants underwent clinical measurements at the hospital in April 2007. 5811 police officers participated in the follow-up consisting of new-onset diabetes (NOD) events occurring annually between 2008 and 2011.

Primary outcome measures: Baseline data were collected from policemen who completed the Symptom Checklist 90-Revised (SCL-90-R) questionnaire and a self-designed questionnaire. Psychological distress was measured by the SCL-90-R questionnaire. Hong Kong Chinese Diabetes Risk Score (HKCDRS) was used to evaluate the risk of T2DM, and the participants were divided into low-risk group and high-risk group based on the HKCDRS. Cox proportional hazards regression was used to calculate the HRs of the incidence of T2DM related to psychological distress and further stratified the analysis based on HKCDRS.

Results: Among 5811 participants, 179 subjects developed NOD during the 4-year follow-up. 54 subjects $(1.63 \%)$ with a HKCDRS $0-7$ vs 125 subjects $(4.98 \%)$ with a HKCDRS $>7$ developed NOD $(p<0.05)$.

There was a significant association between psychological distress and T2DM (HR=1.46; $95 \% \mathrm{Cl}$ 1.05 to 2.02). Among the participants with a high-risk score (HKCDRS>7), $7.07 \%$ of those with psychological distress developed T2DM compared with $4.43 \%$ of participants without psychological distress $(p<0.05)$. The corresponding adjusted HR for psychological distress was 1.61 (95\% $\mathrm{Cl} 1.10$ to 2.37 ).

Conclusions: Psychological distress is an independent risk factor for T2DM in this prospective cohort study. Stratification analysis indicated that psychological distress was associated with T2DM in a high-risk level population.

\section{INTRODUCTION}

Type 2 diabetes mellitus (T2DM) is a complex metabolic disease characterised by

\section{Strengths and limitations of this study}

- This study has evaluated police officers' physical and mental health conditions by clinical measurement and questionnaire survey in a large size of occupational population samples.

- This study has a high response rate $(93 \%)$ and few subjects were lost to follow-up.

- This study has examined whether the association between psychological distress and type 2 diabetes mellitus was different in high-risk versus low-risk populations, which have been scarcely studied among Chinese police officers.

- The effects of psychological distress on prediabetes were failed to consider.

impaired $\beta$-cell dysfunction or insulin resistance. ${ }^{1}$ The hypothalamic-pituitary-adrenal axis and the central sympathetic system are thought to contribute to the metabolic derangement leading to T2DM. ${ }^{2}$ The prevalence of diabetes mellitus is increasing worldwide. The International Diabetes Federation estimated a global prevalence of 382 million people with diabetes in 2013, which is expected to increase to 592 million by $2035 .^{3}$ Compared with developed countries, the incidence of diabetes mellitus is higher in developing countries, India and China have the greatest number of people with diabetes. ${ }^{4}$ As a result of this increasing prevalence, T2DM presents a tremendous clinical and economic burden globally and has become a key public health issue. ${ }^{5}$

Many studies have shown that traditional risk factors such as age, unhealthy habits, obesity, high-fat diet, smoking and excessive alcohol intake are associated with the development of T2DM. ${ }^{6-8}$ However, more recent evidence suggests that psychosocial distress also plays an important role. ${ }^{9}{ }^{10}$ Previous studies have reported that psychological distress can be a cause of insulin resistance, ${ }^{11}$ and that treatment of psychological factors such as depression can potentially improve 
glycemic control. ${ }^{12}{ }^{13}$ Confirmation of this association could have profound implications for the prevention and treatment of these disorders. However, there have been few prospective studies conducted that had evaluated the relationship between psychological factors and T2DM. Furthermore, the findings from these studies are inconsistent.

The association between diabetes and psychological distress is complex, and a simple pattern of cause and effect is not easily discerned. Current studies have focused on the relationship between traditional factors or psychological distress and T2DM. Fewer studies have examined whether these associations with T2DM differ among individuals belonging to a high-risk or low-risk group. The relationship between psychological distress and T2DM has not been well documented among different diabetes risk levels in Chinese policemen, as well. Furthermore, policemen as a special occupational group face more pressure and have a good compliance compared with general population. We chose policemen as our study population to investigate whether psychological factors are associated with chronic disease. Our team has analysed the relationship between psychological distress and dyslipidaemia, clarified the relationship between occupational stressors and the incidence of T2DM based on police officers. ${ }^{14}{ }^{15}$ In this study, we examined (1) whether psychological distress was an independent risk factor for the development of T2DM in a cohort of Chinese police officers and (2) whether psychological distress at baseline differentially predicted the development of T2DM when data were stratified by T2DM risk levels based on the Hong Kong Chinese Diabetes Risk Score (HKCDRS). ${ }^{16}$

\section{STUDY POPULATION AND METHOD}

Police officers, aged 20-60 years, were recruited to participate in the current study between 2007 and 2011 in Tianjin, China. ${ }^{1415}$ A total of 6559 police officers volunteered to participate. Details of the study characteristics are shown in figure 1. At the time of enrolment in 2007, all of the participants had their health status assessed at the Medical Center of Police Hospital and were asked to complete the Symptom Checklist 90-Revised (SCL-90-R) and self-designed questionnaires. The HKCDRS was calculated based on the results of the physical examination and the self-designed questionnaires. Exclusion criteria were the use of insulin or oral hypoglycaemic agents, previously diagnosed diabetes and incomplete information regarding socio-demographic characteristics and mental health status. Finally, the follow-up population consisted of 5811 participants. A clinical examination was performed yearly, and the number of new cases of diabetes between 2008 and 2011 was recorded.

\section{Ethics}

All participants provided informed consent which was provided according to the Declaration of Helsinki.

\section{Measurements of psychological distress}

Psychological distress was assessed by the SCL-90-R, a self-assessment instrument designed to screen for a broad range of psychological disorders. ${ }^{17}$ SCL-90-R includes 90 items that reflect 10 different psychological dimensions: somatisation, obsessive-compulsive, interpersonal sensitivity, depression, anxiety, hostility, phobic anxiety, paranoid ideation, psychoticism and a rest subscale. Each item is rated on a 5-point scale (1-5) of distress. The participant selects one response from the following range of responses: $1=$ not at all; $2=\mathrm{a}$ little bit; $3=$ moderately; $4=$ quite a bit and $5=$ extremely. ${ }^{18}$ The SCL-90-R was used to clarify specific aspects of a psychological problem and/or psychiatric disturbance.

The Chinese version of SCL-90-R has been used among diverse populations ${ }^{19} 20$ and, at the time of enrolment, was administered to assess the participant's psychological status. The questionnaire's internal consistency was satisfactory with a Cornbrash's $\alpha$ of 0.987 and a Guttmann split-half of 0.961 according to our previous report. ${ }^{15}$ The total score (TOS, range 90-450) was calculated by adding the scores of the subscales. The items relevant to each subscale were means of the total scores for all of the questions related to the symptom. According to the baseline score of the SCL-90-R, participants with TOS that exceeded 160 were classified as mentally unhealthy. ${ }^{21}$

\section{Hong Kong Chinese Diabetes Risk Score}

The diabetes risk score was developed as a feasible way of identifying people at risk of developing diabetes to initiate preventive measures. However, differences in ethnicity and cultural factors called for the development of population-relevant risk scores. In this study, we used the diabetes risk score model based on the Hong Kong Chinese study. ${ }^{16}$ A risk score system was derived using the $\beta$ values of the corresponding predictors in the logistic regression analysis. The scoring system consisted of five items: hypertension, age, family history of diabetes, body mass index (BMI) and dyslipidaemia. We labelled the model the HKCDRS. The area under the receiver operator characteristic curve was 0.67 .

\section{Measurements of covariates}

The survey collected the following information: (1) socio-demographic characteristics including age, educational level, marital status, smoking status, alcohol intake, physical activity level, waist circumference, family history of diabetes and dyslipidaemia. These data were obtained from the self-designed questionnaire; (2) clinical characteristics such as body weight, height, blood pressure, fasting blood glucose and blood lipid levels. Age was divided into three groups (20-39, 40-49 and $50-60$ years). A family history of diabetes was defined as having at least one first-degree relative (parents, siblings) or two second-degree relatives (grandparents, uncles or aunts) with diabetes and was categorised as positive or negative. ${ }^{11}$ Smoking habits were categorised 


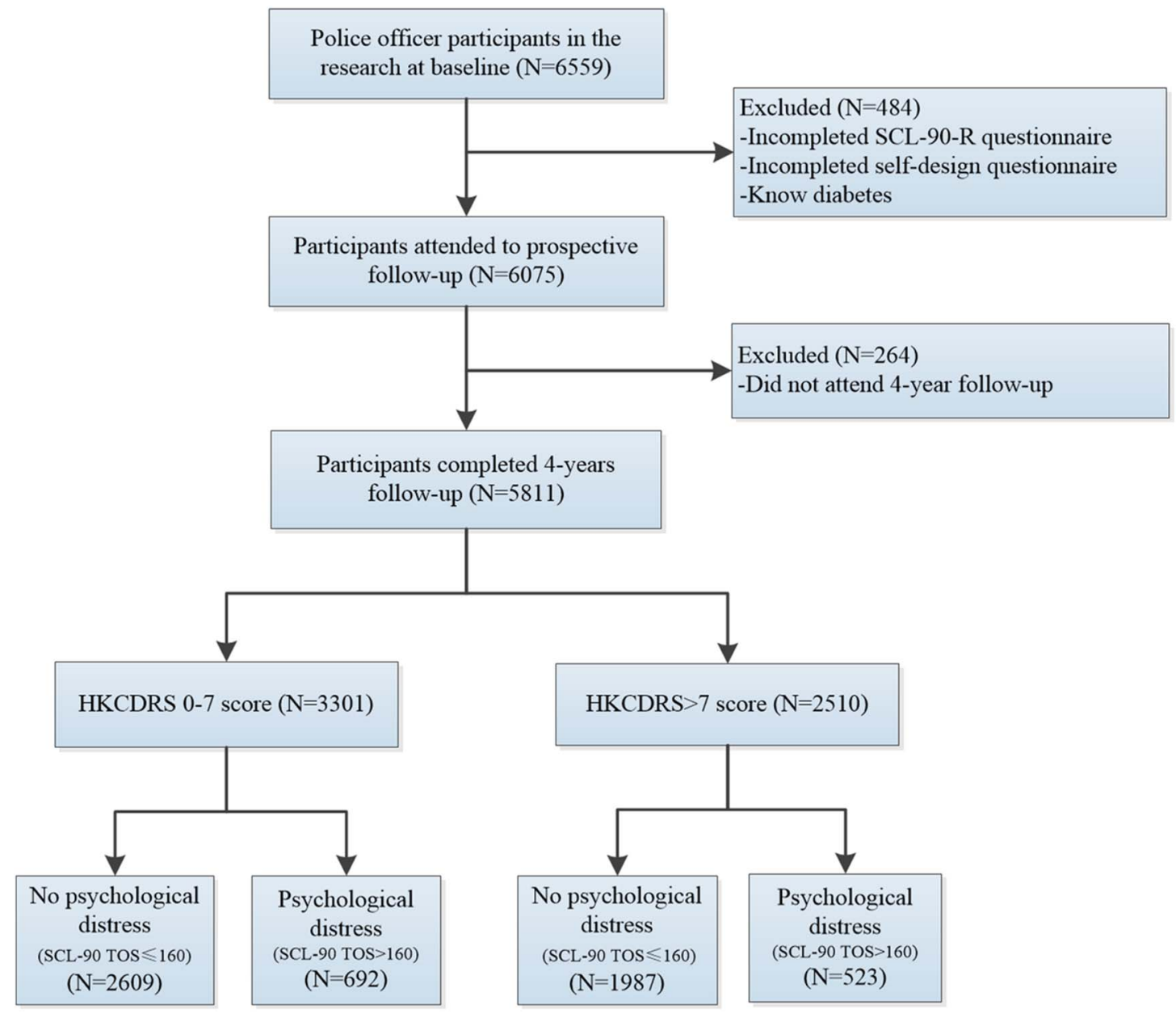

Figure 1 Definition of the study participants. A total of 5811 participants who completed a 4-year follow-up were divided into four groups according to the HKCDRS and psychological distress status. HKCDRS, Hong Kong Chinese Diabetes Risk Score; TOS, total score.

as non-smoker, ex-smoker and current smoker, and alcohol consumption was grouped into current drinker, former drinker and never drinker. The BMI was calculated as the weight in kilograms divided by the height in metres squared ${ }^{22}$ and was subsequently divided into three groups $\left(<25,25-29.99\right.$ and $\left.\geq 30 \mathrm{~kg} / \mathrm{m}^{2}\right)$. Exercise habits were split into never (less than once per week), occasionally (one or two times per week) and often (more than three times per week).

\section{Laboratory assay}

An overnight fasting venous blood sample was taken between 08:00 and noon and transported to the Medical Center of Police Hospital. Fasting plasma glucose (FPG), blood lipid levels, insulin, serum uric acid, serum urea nitrogen and serum creatinine were measured according to standardised methods. The $75 \mathrm{~g}$ oral glucose tolerance test (OGTT) was performed in accordance with the WHO criteria. Blood pressure in the left upper arm was measured three times with a mercury sphygmomanometer after $5 \mathrm{~min}$ of rest, and the average was calculated.

\section{Diagnosis of T2DM}

T2DM was diagnosed according to the American Diabetes Association diagnostic criteria when any one of the following symptoms was observed: ${ }^{23}$ (1) $\mathrm{FPG} \geq 7.0 \mathrm{mmol} / \mathrm{L}$ $(126 \mathrm{mg} / \mathrm{dL})$; (2) an OGTT $\geq 11.1 \mathrm{mmol} / \mathrm{L}(200 \mathrm{mg} / \mathrm{dL})$; (3) random blood glucose $\geq 11.1 \mathrm{mmol} / \mathrm{L}$ (200 mg/dL); (4) using insulin or oral hypoglycaemic agents.

\section{Statistical analyses}

The continuous and categorical variables were presented as the mean and $\mathrm{SD}($ mean $\pm \mathrm{SD})$ and frequencies, respectively. For the comparison of characteristics between participants at baseline with or without the onset of diabetes, we used the $\chi^{2}$ test for categorical variables and the independent sample t-test for continuous variables. Comparison of the incidence of new-onset diabetes (NOD) and non-diabetes (ND) was conducted using the Cox proportional hazards regression to calculate the HR and accompanying 95\% CI. The HR and 95\% CI were computed to compare the effects of traditional risk factors on the incidence of diabetes and were adjusted for covariates including age, smoking status, 
alcohol intake, education level, physical level, marital status, BMI, family history of diabetes, blood pressure, blood lipids and police classification.

The participants were divided into two groups based on the HKCDRS (score of $0-7$, score $\geq 8$ ). The score of 7 was determined as the cut-off based on the Youden index. Participants were grouped into four groups according to the level of psychological distress (shown in the flow chart, figure 1): (1) HKCDRS 0-7, no psychological distress; (2) HKCDRS 0-7, psychological distress; (3) HKCDRS $>7$, no psychological distress; (4) HKCDRS $>7$, psychological distress. The Cox proportional hazards regression was used among the four groups after adjustment for marital status, blood pressure, blood lipids, educational level and police classification.

Statistical analysis was performed using the Statistical Package for the Social Sciences (SPSS) for Windows (V.18.0, Chicago, Illinois, USA). $p$ Values of $<0.05$ were considered statistically significant, and all $\mathrm{p}$ values were two-sided.

\section{RESULTS}

Population characteristics

Table 1 shows the baseline characteristics of the policemen according to the occurrence of T2DM. Participants with T2DM during follow-up were older, with a greater

Table 1 Baseline demographic, lifestyle, HKCDRS, working conditions and psychological distress characteristics of subjects according to the occurrence of T2DM

\begin{tabular}{|c|c|c|c|c|}
\hline & Non-diabetes $(n=5632)$ & New-onset diabetes $(n=179)$ & $\chi^{2} / \mathrm{t}$ & p Value \\
\hline Age (years) & $37.27 \pm 9.01$ & $42.66 \pm 9.21$ & 7.764 & $<0.001$ \\
\hline $\mathrm{BMI}\left(\mathrm{kg} / \mathrm{m}^{2}\right)$ & $25.77 \pm 3.34$ & $26.97 \pm 3.54$ & 4.672 & $<0.001$ \\
\hline SCL-90-R score & $134.56 \pm 46.80$ & $142.23 \pm 50.22$ & & 0.031 \\
\hline Marital status (n, \%) & & & 0.742 & 0.690 \\
\hline Married & $5214(92.6)$ & $165(92.2)$ & & \\
\hline Other & $418(7.4)$ & $2(7.8)$ & & \\
\hline Education level (n, \%) & & & 4.396 & 0.041 \\
\hline College and above & $5491(97.5)$ & $170(95.0)$ & & \\
\hline Less than college & $141(2.5)$ & $9(5.0)$ & & \\
\hline Exercise activity (n, \%) & & & 2.292 & 0.318 \\
\hline Often (more than three times per week) & $1228(21.8)$ & $31(17.3)$ & & \\
\hline Occasion & $3747(66.5)$ & $128(71.5)$ & & \\
\hline Never & $657(11.7)$ & 20 (11.2) & & \\
\hline Smoking (n, \%) & & & 1.022 & 0.600 \\
\hline Non-smokers & $1808(32.1)$ & $53(29.6)$ & & \\
\hline Current smokers & $3218(57.1)$ & $109(60.9)$ & & \\
\hline Ex-smokers & $606(10.8)$ & $17(9.5)$ & & \\
\hline Alcohol intake $(\mathrm{n}, \%)$ & & & 3.348 & 0.188 \\
\hline Never or almost never & $432(7.7)$ & $13(7.3)$ & & \\
\hline Current using alcohol & $5068(90.0)$ & $158(88.3)$ & & \\
\hline Former using alcohol & $132(3.3)$ & $8(4.5)$ & & \\
\hline Hypertension (n, \%) & & & 24.000 & $<0.001$ \\
\hline No & $3986(70.8)$ & $95(53.1)$ & & \\
\hline Yes & $1646(29.2)$ & 84 (46.9) & & \\
\hline Dyslipidaemia (n, \%) & & & 14.037 & $<0.001$ \\
\hline No & 3337 (59.3) & $81(45.3)$ & & \\
\hline Yes & $2295(40.7)$ & $98(54.7)$ & & \\
\hline Family history of diabetes (n, \%) & & & 0.157 & 0.733 \\
\hline No & 4109 (73.0) & $133(74.3)$ & & \\
\hline Yes & $1523(27.0)$ & $46(25.7)$ & & \\
\hline HKCDRS (n, \%) & & & 65.844 & $<0.001$ \\
\hline $0-7$ scores & $3427(57.7)$ & $54(30.2)$ & & \\
\hline$>7$ scores & 2385 (42.3) & $125(69.8)$ & & \\
\hline Police assignments ( $\mathrm{n}, \%)$ & & & 97.332 & $<0.001$ \\
\hline Criminal investigation & $737(13.1)$ & $11(6.1)$ & & \\
\hline Public security & $1484(26.3)$ & $25(14.0)$ & & \\
\hline Administrative services & $768(13.6)$ & $12(6.7)$ & & \\
\hline Traffic control & 1477 (26.2) & $106(59.2)$ & & \\
\hline Household registration & $799(14.2)$ & $14(7.8)$ & & \\
\hline Other & $367(6.5)$ & $11(6.1)$ & & \\
\hline
\end{tabular}

BMI, body mass index; HKCDRS, Hong Kong Chinese Diabetes Risk Score; SCL-90-R, Symptom Checklist 90-Revised; T2DM, type 2 diabetes mellitus. 
BMI and a higher SCL-90-R score compared with participants without diabetes. The incidence of hypertension, dyslipidaemia, HKCDRS and police assignment differed significantly between NOD and ND groups (table 1). However, smoking status, alcohol intake, physical activity and a family history of diabetes were not significantly different between the groups.

\section{Incidence of type 2 diabetes mellitus}

Among 5811 participants, 179 subjects developed NOD during the 4-year follow-up. The incidence (the number of new cases) of NOD was 34 in 2008, 57 in 2009, 30 in 2010 and 58 in 2011, the annual incidence rate was 9.5/ 100 person-years. Figure 2 shows the unadjusted incidence, 54 subjects $(1.63 \%)$ with a HKCDRS $0-7$ vs 125 subjects (4.98\%) with a HKCDRS $>7$ developed NOD $(\mathrm{p}<0.05)$. Among the participants with a HKCDRS of 0 $7,2.02 \%$ of those with psychological distress and $1.53 \%$ of those without psychological distress developed T2DM during the follow-up period. The level of psychological distress did not differ in the groups that were at a low risk for the development of T2DM. However, among the participants with HKCDRS $>7,7.03 \%$ of those with psychological distress developed T2DM compared with $4.43 \%$ of the participants without psychological distress $(\mathrm{p}<0.05)$.

\section{Multivariate analyses of HKCDRS and psychological distress}

In the multivariate Cox proportional hazards regression, after adjustments of the covariates, there was a significant association between psychological distress and T2DM (HR=1.46; 95\% CI 1.05 to 2.02) (table 2). Results

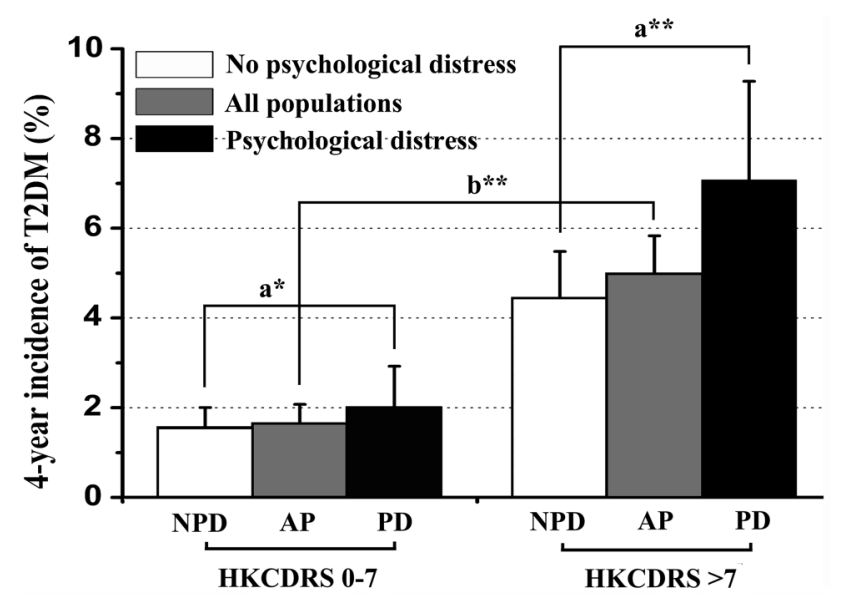

Figure 2 Unadjusted 4-year incidence $(95 \% \mathrm{Cl})$ of T2DM among participants in different HKCDRS groups. The participants were further stratified by the psychological distress status. Unadjusted incidence of participants with psychological distress in HKCDRS of $>7$ group was higher than participants without psychological distress $(p<0.05)$. AP, all populations; HKCDRS, Hong Kong Chinese Diabetes Risk Score; NPD, no psychological distress; PD, psychological distress. $a^{\star}: p>0.05 ; b^{\star \star}: p<0.05 ; a^{\star \star}: p<0.05$. of the multivariable Cox proportional hazards regression confirm a strong association between HKCDRS and the incidence of T2DM. Among subjects without psychological distress, high-risk group (HKCDRS $>7$ ) has a higher risk of diabetes compared with low-risk group $(\mathrm{HKCDRS}<7) \quad(\mathrm{HR}=2.66,95 \%$ CI 1.82 to 3.88$)$ in comparison 1 (table 3). Similar results were observed among subjects with psychological distress in comparison 2 (table 3), a significant difference in the association between the HKCDRS and the incidence of T2DM (HR=3.36, 95\% CI 1.81 to 6.23). Thus, high diabetes risk score is an independent risk factor for diabetes.

We then evaluated whether psychological distress is associated with the incidence of T2DM in two HKCDRS groups. Table 3 shows the association between the combination of the HKCDRS and psychological distress and the risk of T2DM. Comparison 3 indicated that among subjects with a HKCDRS $>7$, the presence of psychological distress was associated with an increased risk of T2DM (HR $=1.61,95 \%$ CI 1.10 to 2.37$)$. However, when compared with subjects with a HKCDRS of $0-7$ in comparison 1, with no psychological distress, the HR was 1.28 (95\% CI 0.69 to 2.35 ).

We used a subgroup Cox regression analysis among traffic control police officers to control for the effects of environmental risk factors. Psychological distress in these officers increased their risk of developing T2DM compared with subjects in a high-risk group without psychological distress (HR=1.84, 95\% CI 1.06 to 3.17) (table 4). These results were consistent across all populations and indicated that psychological distress predicted the incidence of T2DM in subjects with a high HKCDRS.

\section{DISCUSSION}

In this study, we examined whether the presence of psychological distress at baseline was an independent risk factor for the development of T2DM, and whether the presence of psychological distress differentially predicted the incidence of T2DM at different risk levels according to the HKCDRS. Our main findings were that (1) there was a significant association between psychological distress and T2DM in a multivariate model during a 4-year follow-up of a cohort of Chinese police officers, and (2) psychological distress was associated with a 1.61-fold increase in the risk of T2DM in subjects with a high HKCDRS. These results indicate that psychological distress primarily contributes to the development of T2DM in a high-risk population.

Previous studies have suggested that psychological distress significantly increases the risk of T2DM. ${ }^{24-26}$ In this study, we discovered that psychological distress plays an important role in the development of T2DM. However, initially we found no overall relationship between psychological distress and T2DM in groups with the same HKCDRS. A stratified analysis also revealed no association between psychological distress and T2DM in low-risk subjects compared with participants without 
Table 2 Cox regression analyses of the association between psychological distress, traditional risk factors and T2DM

\begin{tabular}{|c|c|c|c|}
\hline & HR & $95 \% \mathrm{Cl}$ & p Value \\
\hline Age (years) & & & $<0.001$ \\
\hline$<40$ & Reference & & \\
\hline $40-50$ & 1.92 & 1.35 to 2.75 & $<0.001$ \\
\hline$\geq 50$ & 2.36 & 1.56 to 3.57 & $<0.001$ \\
\hline $\mathrm{BM}\left(\mathrm{kg} / \mathrm{m}^{2}\right)$ & & & 0.132 \\
\hline$<25$ & Reference & & \\
\hline $25-30$ & 1.35 & 0.94 to 1.93 & 0.102 \\
\hline$\geq 30$ & 1.60 & 0.98 to 2.63 & 0.061 \\
\hline Marital status & 1.34 & 0.77 to 2.32 & 0.294 \\
\hline Education level & 1.40 & 0.71 to 2.75 & 0.337 \\
\hline Exercise activity & & & 0.184 \\
\hline Often (more than three times per week) & Reference & & \\
\hline Occasion & 1.43 & 0.96 to 2.13 & 0.079 \\
\hline Never & 1.17 & 0.66 to 2.08 & 0.585 \\
\hline Smoking & & & 0.653 \\
\hline Non-smokers & Reference & & \\
\hline Current smokers & 1.02 & 0.73 to 1.43 & 0.889 \\
\hline Ex-smokers & 0.80 & 0.46 to 1.40 & 0.438 \\
\hline Alcohol intake & & & 0.464 \\
\hline Never or almost never & Reference & & \\
\hline Current using alcohol & 1 & 0.56 to 1.77 & 0.988 \\
\hline Former using alcohol & 1.57 & 0.64 to 3.84 & 0.324 \\
\hline Hypertension & 1.60 & 1.17 to 2.20 & 0.003 \\
\hline Dyslipidaemia & 1.41 & 1.04 to 1.92 & 0.027 \\
\hline Family history of diabetes & 1.04 & 0.74 to 1.45 & 0.84 \\
\hline Working hours & 1.08 & 0.80 to 1.47 & 0.618 \\
\hline Police assignments & & & $<0.001$ \\
\hline House registration & Reference & & \\
\hline Public security & 0.96 & 0.50 to 1.85 & 0.894 \\
\hline Administrative services & 0.84 & 0.39 to 1.85 & 0.672 \\
\hline Traffic control & 3.35 & 1.89 to 5.93 & $<0.001$ \\
\hline Criminal investigation & 0.88 & 0.40 to 1.95 & 0.757 \\
\hline Other & 1.45 & 0.65 to 3.21 & 0.362 \\
\hline Psychological distress & 1.46 & 1.05 to 2.02 & 0.024 \\
\hline
\end{tabular}

BMI, body mass index; T2DM, type 2 diabetes mellitus.

psychological distress. The HR was 1.28 (95\% CI 0.69 to 2.35 ) and the difference was not significant. However, in the high HKCDRS subgroup, we observed a 1.61-fold increase in the risk of T2DM in subjects with psychological distress compared with those without psychological distress. In the absence of traditional risk factors, psychological distress may be a primary causative agent in the development of T2DM. Consistent with our results, Marianna et al concluded that psychological distress was associated with an accelerated progression to T2DM in individuals with a higher Framingham Offspring Type 2 Diabetes Risk Score. ${ }^{27}$

The debate about the relationship between psychological distress and T2DM is still ongoing. In the Nurses' Health Study II, occupational distress was not correlated with self-reported T2DM. A series of Whitehall II studies $^{28} 29$ and two studies from Sweden suggested that work-related stress increased the risk of T2DM in women, but not in men. ${ }^{30}{ }^{31}$ A German study of men working in industry found that psychological distress was associated with diabetes. ${ }^{32}$ 33 In addition, Norito Kawakami demonstrated that psychological working conditions were a risk factor for the development of non-insulin-dependent diabetes mellitus in Japanese men. ${ }^{33}$ Current studies have provided confirmatory evidence that psychosocial distress was a potential risk factor for T2DM. ${ }^{34}$ In line with these results, we found a significant relationship between psychological distress and T2DM (HR=1.46; 95\% CI 1.05 to 2.02) after multivariate adjustments. The controversy in the literature surrounding the link between psychological stress and diabetes may in part be due to the use of different psychological distress measurement scales and diagnostic criteria for diabetes.

Depression has been shown to be twice as common in people with T1DM and T2DM as in the general population, affecting $10-20 \%$ of adults with diabetes. ${ }^{35}$ Researchers suspect that the link between T2DM and depression is in fact a two-way street, ${ }^{36}{ }^{37}$ and evidence supports the presence of biological correlates of depression in patients with T2DM. A state of high stress may lead to unhealthy lifestyles, ${ }^{38}{ }^{39}$ such as high-fat diet, 
Table 3 Cox regression analyses of the association between psychological distress and T2DM among different HKCDRS groups

\section{HKCDRS and psychological distress at baseline}

Sample size

HR $(95 \% \mathrm{Cl})$ for 4-years incident T2DM

HKCDRS 0-7, no psychological distress

HKCDRS 0-7, psychological distress

$2609 \quad 40$

HKCDRS $>7$, no psychological distress

1987

523

Comparison

Comparison 2

Comparison 3

Comparison 4

HKCDRS $>7$, psychological distress

14

Reference

0.78 (0.43 to 1.44$)$

$0.48(0.27$ to 0.85$)$

$0.30(0.16$ to 0.55$)$

Reference

$0.38(0.26$ to 0.55$)$

$0.62(0.42$ to 0.91$)$

2.66 (1.82 to 3.88$) \quad 2.08$ (1.18 to 3.67$) \quad$ Reference

$0.23(0.15$ to 0.37$)$

assignments. All groups are shown in comparisons 1-4. Models are adjusted for marital

assignments. All comparisons are based on the same data, but with a different reference
HKCDRS, Hong Kong Chinese Diabetes Risk Score; T2DM, type 2 diabetes mellitus.

Table 4 Cox regression analyses of the association between psychological distress and T2DM among different HKCDRS groups in traffic police officers

\begin{tabular}{|c|c|c|c|c|c|c|}
\hline \multirow[b]{2}{*}{ HKCDRS and psychological distress at baseline } & \multirow[b]{2}{*}{ Sample size } & \multirow[b]{2}{*}{ Cases } & \multicolumn{4}{|c|}{ HR (95\% Cl) for 4-year incidence of T2DM } \\
\hline & & & Comparison 1 & Comparison 2 & Comparison 3 & Comparison 4 \\
\hline HKCDRS 0-7, no psychological distress & 632 & 24 & Reference & 1.75 (0.53 to 5.77$)$ & 0.26 (0.08 to 0.83 ) & $0.14(0.04$ to 0.48$)$ \\
\hline HKCDRS 0-7, psychological distress & 174 & 7 & 0.57 (0.17 to 1.88$)$ & Reference & 0.45 (0.29 to 0.71$)$ & $0.54(0.32$ to 0.94$)$ \\
\hline HKCDRS $>7$, no psychological distress & 620 & 56 & 2.20 (1.40 to 3.47$)$ & $3.86(1.21$ to 12.35$)$ & Reference & $0.25(0.13$ to 0.45$)$ \\
\hline HKCDRS>7, psychological distress & 157 & 19 & 4.05 (2.20 to 7.44$)$ & 7.09 (2.07 to 24.29$)$ & $1.84(1.06$ to 3.17$)$ & Reference \\
\hline
\end{tabular}

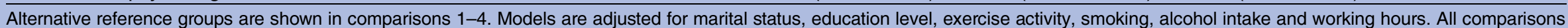
are based on the same data, but with a different reference group.

HKCDRS, Hong Kong Chinese Diabetes Risk Score; T2DM, type 2 diabetes mellitus. 
smoking, alcohol intake, low physical activity, which may in turn affect HbA1c levels. ${ }^{40}$

In this study, more than $50 \%$ of the NOD cases occurred in the group of traffic control police officers $(\mathrm{HR}=3.35) \quad$ (tables 1 and 2). The baseline of traditional risk factors for T2DM, BMI, smoking status, alcohol intake, physical activity and other traditional risk factors were not significantly different between the police assignments (see online supplementary table S1) except the constituent ratio of age. Traffic police officers spend a great deal of time working outdoors in China and are constantly exposed to environmental risk factors such as $\mathrm{PM}_{2.5}$, automobile exhaust and traffic noise. Studies have shown that environmental risk factors increased the incidence of T2DM. ${ }^{41-43}$ In a pooled meta-analysis, there was a $10 \%$ increased risk of T2DM per $10 \mathrm{mg} / \mathrm{m}^{3}$ increase in exposure to fine particulate matter $\left(\mathrm{PM}_{2.5}\right)$ and an $8 \%$ increased risk of T2DM increased per $10 \mathrm{mg} / \mathrm{m}^{3}$ exposure to nitrogen dioxide. ${ }^{44}$ Long-term exposure to total PM increased T2DM risk in the general population, and local traffic-specific PM was related to higher risks of T2DM than the total PM. ${ }^{45}$ However, we have not conducted a panel study to evaluate personal air pollution exposure. In order to certificate psychological distress was associated with T2DM in traffic police officers with high diabetes risk, a subgroup Cox regression analysis among traffic police subjects was conducted. In the subgroup analysis, we observed a 1.84-fold increased risk of T2DM associated with psychological distress in traffic police officers with a high HKCDRS. The external environment, including noise, air pollution, cold and heat, has direct and indirect effects on T2DM. For example, severe conditions in the external environment may contribute to psychological distress. We have previously found that the physical environment functions as an occupational stressor is associated with the development of T2DM. ${ }^{15}$

One plausible mechanism by which psychological distress may predispose to T2DM is through stimulation of the sympathetic nervous system and pituitary gland activity ${ }^{46} 47$ that leads to a rise in circulating catabolic hormones and suppression of anabolic hormone levels. ${ }^{48}$ These neuroendocrine systems are important regulators of glucose uptake, release and storage. The stress response is known to increase cortisol secretion, ${ }^{49}$ which stimulates glucose production in the liver and antagonises the action of insulin in peripheral tissues. This neuroendocrine profile has been associated with abdominal adiposity and increased triglyceride and insulin levels, all of which are established predictors of T2DM. Repeated or continuous activation of these systems during stress, characterised as a paradoxical state of high metabolic activity without corresponding metabolic needs, might predispose to the development of diabetes.

\section{Limitations}

There were several limitations to our study that could influence our results. First, we failed to consider the effects of psychological distress on pre-diabetes, a highrisk condition for the development of diabetes, which can last 10 years. ${ }^{50}$ Second, the SCL-90-R instrument was widely applicated in many studies, but a number of studies have reported the psychometric weaknesses of the SCL-90-R, a central criticism is the postulated factor structure was not replicable in our study. Third, a subgroup analysis indicated that psychological distress increased the incidence of T2DM among traffic police subjects. However, traffic police officers generally work outdoors; we have considered the interaction between external environment and psychological distress, but we have not conducted a panel study to evaluate personal air pollution exposure, which could also potentially affect the progression of diabetes mellitus.

\section{CONCLUSION}

In conclusion, this observational study found that psychological distress was an independent risk factor for the development of T2DM. The impact of psychological distress on the development of T2DM has healthcare implications that should encourage us to focus on vulnerable populations that are exposed to psychological distress, especially with those belonging to a high-risk group for T2DM.

\section{Author affiliations}

${ }^{1}$ Department of Occupational and Environmental Health, School of Public Health, Tianjin Medical University, Tianjin, China

${ }^{2}$ Tongling University, Tongling, Anhui, China

${ }^{3}$ Department of Cardiology, Tianjin $4^{\text {th }}$ Center Hospital, The $4^{\text {th }}$ Center

Hospital of Tianjin Medical University, Tianjin, China

${ }^{4}$ Tianjin Emergency Medical Center, Tianjin, China

${ }^{5}$ Medical Center of Police Hospital, Tianjin, China

${ }^{6}$ Tianjin Centers for Disease Control and Prevention, Tianjin, China

Acknowledgements We thank all those involved in the research project.

Contributors NJT and FST have received research support from the Tianjin Municipal Science and Technology Commission. CL, JCL, XX, SY, XC and SY have collected the research materials and analysed the data. CL, JCL and NJT have written, reviewed or approved the submitted manuscript. All of the authors have participated actively in the study and have read and approved the submitted manuscript.

Funding The work was supported from the Tianjin scientific and technological support key projects (grant numbers 08ZCGYSF01500 and 10ZCKFSF00600), Tianjin Municipal Science and Technology Commission.

Competing interests None declared.

Patient consent Obtained.

Ethics approval The protocol was approved by the Ethics Committee of Tianjin Medical University (Tianjin, China).

Provenance and peer review Not commissioned; externally peer reviewed.

Data sharing statement No additional data are available.

Open Access This is an Open Access article distributed in accordance with the terms of the Creative Commons Attribution (CC BY 4.0) license, which permits others to distribute, remix, adapt and build upon this work, for commercial use, provided the original work is properly cited. See: http:// creativecommons.org/licenses/by/4.0/

\section{REFERENCES}

1. Jeffery $N$, Harries LW. Beta cell differentiation status in type 2 diabetes. Diabetes Obes Metab 2016;18:1167-75. 
2. Kyrou I, Chrousos GP, Tsigos C. Stress, visceral obesity, and metabolic complications. Ann N Y Acad Sci 2006;1083:77-110.

3. Guariguata L, Whiting DR, Hambleton I, et al. Global estimates of diabetes prevalence for 2013 and projections for 2035. Diabetes Res Clin Pract 2014;103:137-49.

4. Ramachandran A, Ma RC, Snehalatha C. Diabetes in Asia. Lancet 2010;375:408-18.

5. Laura A, Caraveo J, Patricia B, et al. Cross-national comparisons of the prevalences and correlates of mental disorders. WHO International Consortium in Psychiatric Epidemiology. Bull World Health Organ 2000;78:413-26.

6. Al-Goblan AS, Al-Alfi MA, Khan MZ. Mechanism linking diabetes mellitus and obesity. Diabetes Metab Syndr Obes 2014;7:587-91.

7. Boyle T, Fritschi L, Tabatabaei SM, et al. Smoking, alcohol, diabetes, obesity, socioeconomic status, and the risk of colorectal cancer in a population-based case-control study. Cancer Causes Control 2014;25:1659-68.

8. Oba S, Suzuki E, Yamamoto M, et al. Active and passive exposure to tobacco smoke in relation to insulin sensitivity and pancreatic beta-cell function in Japanese subjects. Diabetes Metab 2015;41:160-7.

9. Djindjic N, Jovanovic J, Djindjic B, et al. Associations between the occupational stress index and hypertension, type 2 diabetes mellitus, and lipid disorders in middle-aged men and women. Ann Occup Hyg 2012;56:1051-62.

10. Kamble SV, Phalke DB. Study of occupational stress as a risk factor for various morbidities among policemen. $J$ Indian Med Assoc 2011;109:238-40.

11. Agardh EE, Ahlbom A, Andersson T, et al. Work stress and low sense of coherence is associated with type 2 diabetes in middle-aged Swedish women. Diabetes Care 2003;26:719-24.

12. Lustman PJ, Griffith LS, Freedland KE, et al. Cognitive behavior therapy for depression in type 2 diabetes mellitus. A randomized, controlled trial. Ann Intern Med 1998;129:613-21.

13. Lustman PJ, Freedland KE, Griffith LS, et al. Fluoxetine for depression in diabetes: a randomized double-blind placebo-controlled trial. Diabetes Care 2000;23:618-23.

14. Chen X, Leng L, Yu H, et al. Psychological distress and dyslipidemia in Chinese police officers: a 4-year follow-up study in Tianjin, China. $J$ Occup Environ Med 2015;57:400-5.

15. Yu H, Liu JC, Fan YJ, et al. Association between occupational stressors and type 2 diabetes among Chinese police officers: a 4-year follow-up study in Tianjin, China. Int Arch Occup Environ Health 2016;89:277-88.

16. Ko G, So W, Tong $P$, et al. A simple risk score to identify Southern Chinese at high risk for diabetes. Diabet Med 2010;27:644-9.

17. Divaris K, Mafla AC, Villa-Torres L, et al. Psychological distress and its correlates among dental students: a survey of 17 Colombian dental schools. BMC Med Educ 2013;13:91.

18. Hoencamp R, Idenburg FJ, van Dongen TT, et al. Long-term impact of battle injuries; five-year follow-up of injured Dutch servicemen in Afghanistan 2006-2010. PLOS ONE 2015;10:e0115119.

19. Yuan KC, Ruo Yao Z, Zhen Yu S, et al. Prevalence and predictors of stress disorders following two earthquakes. Int J Soc Psychiatry 2013;59:525-30.

20. Zhang H, Qian HZ, Meng SQ, et al. Psychological distress, social support and medication adherence in patients with ischemic stroke in the mainland of China. J Huazhong Univ Sci Technol Med Sci 2015;35:405-10.

21. Liu $\mathrm{Y}$, Song $\mathrm{H}$, Wang $\mathrm{T}$, et al. Determinants of tobacco smoking among rural-to-urban migrant workers: a cross-sectional survey in Shanghai. BMC Public Health 2015;15:131.

22. Liu M, Wang JY, Zhu L, et al. Body mass index and serum lipid profile influence serum prostate-specific antigen in Chinese men younger than 50 years of age. Asian J Androl 2011;13:640-3.

23. James R, Gavin III, Mayer B, et al. Report of the expert committee on the diagnosis and classification of diabetes mellitus. Diabetes Care 2003;26(Suppl 1):S5-20.

24. Toshihiro M, Saito K, Takikawa S, et al. Psychosocial factors are independent risk factors for the development of Type 2 diabetes in Japanese workers with impaired fasting glucose and/or impaired glucose tolerance. Diabet Med 2008;25:1211-17.

25. Brunner EJ, Kivimäki M. Epidemiology: work-related stress and the risk of type 2 diabetes mellitus. Nat Rev Endocrinol 2013:9:449-50.

26. Mommersteeg PM, Herr R, Zijlstra WP, et al. Higher levels of psychological distress are associated with a higher risk of incident diabetes during 18 year follow-up: results from the British household panel survey. BMC Public Health 2012;12:1109.
27. Virtanen M, Ferrie JE, Tabak AG, et al. Psychological distress and incidence of type 2 diabetes in high-risk and low-risk populations: the Whitehall II Cohort Study. Diabetes Care 2014;37: 2091-7.

28. Heraclides A, Chandola T, Witte DR, et al. Psychosocial stress at work doubles the risk of type 2 diabetes in middle-aged women: evidence from the Whitehall II study. Diabetes Care 2009;32:2230-5.

29. Heraclides AM, Chandola T, Witte DR, et al. Work stress, obesity and the risk of type 2 diabetes: gender-specific bidirectional effect in the Whitehall II study. Obesity (Silver Spring) 2012;20:428-33.

30. Norberg M, Stenlund H, Lindahl B, et al. Work stress and low emotional support is associated with increased risk of future type 2 diabetes in women. Diabetes Res Clin Pract 2007;76:368-77.

31. Eriksson AK, van den Donk M, Hilding A, et al. Work stress, sense of coherence, and risk of type 2 diabetes in a prospective study of middle-aged Swedish men and women. Diabetes Care 2013;36:2683-9.

32. Li J, Jarczok MN, Loerbroks A, et al. Work stress is associated with diabetes and prediabetes: cross-sectional results from the MIPH Industrial Cohort Studies. Int J Behav Med 2013;20:495-503.

33. Kawakami N, Araki S, Takatsuka N, et al. Overtime, psychosocial working conditions, and occurrence of non-insulin dependent diabetes mellitus in Japanese men. $J$ Epidemiol Community Health 1999;53:359-63.

34. Novak M, Björck L, Giang KW, et al. Perceived stress and incidence of Type 2 diabetes: a 35-year follow-up study of middle-aged Swedish men. Diabet Med 2013;30:e8-16.

35. Anderson RJ, Freedland KE, Clouse RE, et al. The prevalence of comorbid depression in adults with diabetes: a meta-analysis. Diabetes Care 2001;24:1069-78.

36. Mezuk B, Eaton WW, Albrecht S, et al. Depression and type 2 diabetes over the lifespan: a meta-analysis. Diabetes Care 2008;31:2383-90.

37. Golden $\mathrm{SH}$, Lazo M, Carnethon $\mathrm{M}$, et al. Examining a bidirectional association between depressive symptoms and diabetes. JAMA 2008;299:2751-9.

38. McMartin SE, Jacka FN, Colman I. The association between fruit and vegetable consumption and mental health disorders: evidence from five waves of a national survey of Canadians. Prev Med 2013;56:225-30.

39. Weyerer S. Physical inactivity and depression in the community. Evidence from the Upper Bavarian Field Study. Int J Sports Med 1992;13:492-6.

40. Takahashi E, Moriyama K, Yamakado M. Lifestyle and glycemic control in Japanese adults receiving diabetes treatment: an analysis of the 2009 Japan Society of Ningen Dock database. Diabetes Res Clin Pract 2014;104:e50-3.

41. Sade MY, Kloog I, Liberty IF, et al. Air pollution and serum glucose levels: a population-based study. Medicine 2015;94:e1093.

42. Park SK, Adar SD, O'Neill MS, et al. Long-term exposure to air pollution and type 2 diabetes mellitus in a multiethnic cohort. $A m$ J Epidemiol 2015;181:327-36.

43. Rao X, Montresor-Lopez J, Puett R, et al. Ambient air pollution: an emerging risk factor for diabetes mellitus. Curr Diab Rep 2015;15:603.

44. Eze IC, Hemkens LG, Bucher HC, et al. Association between ambient air pollution and diabetes mellitus in Europe and North America: systematic review and meta-analysis. Environ Health Perspect 2015;123:381-9.

45. Weinmayr G, Hennig F, Fuks K, et al. Long-term exposure to fine particulate matter and incidence of type 2 diabetes mellitus in a cohort study: effects of total and traffic-specific air pollution. Environ Health 2015;14:53.

46. Knol MJ, Twisk JW, Beekman AT, et al. Depression as a risk factor for the onset of type 2 diabetes mellitus. A meta-analysis. Diabetologia 2006;49:837-45.

47. Krishnan V, Nestler EJ. The molecular neurobiology of depression. Nature 2008:455:894-902.

48. Alderman SL, McGuire A, Bernier NJ, et al. Central and peripheral glucocorticoid receptors are involved in the plasma cortisol response to an acute stressor in rainbow trout. Gen Comp Endocrinol 2012;176:79-85.

49. Tabák AG, Akbaraly TN, Batty GD, et al. Depression and type 2 diabetes: a causal association? Lancet Diabetes Endocrinol 2014;2:236-45.

50. Tabák AG, Herder C, Rathmann W, et al. Prediabetes: a high-risk state for diabetes development. Lancet 2012;379:2279-90. http://dx. doi.org/10.1016/S0140-6736(12)60283-9 